\title{
Analysis and Test of Hydrodynamic Ram in Welded Metallic Water Tanks
}

\author{
Jong Heon Kim* and Chun-Gon Kim** \\ Department of Aerospace Engineering, Korea Advanced Institute of Science and Technology, Daejeon 305-701, Korea
}

\author{
Seungmoon Jun*** \\ UAV Program Management Office, Agency for Defense Development, Daejeon 305-600, Korea
}

\begin{abstract}
Analysis and test of hydrodynamic ram in welded metallic tanks containing water were performed to investigate the phenomena and to understand the effects on the resulting structural behavior. Arbitrary Lagrange-Euler coupling method was used for the analysis of the fluid-structure interaction occurring in the hydrodynamic ram, where the projectile, tank, and water are exchanging load, momentum, and energy during the traveling of the projectile through the water of the tank. For a better representation of the physical phenomena, modeling of the welded edges is added to the analysis to simulate the earlier weld line fracture and its influence on the resulting hydrodynamic ram behavior. Corresponding hydrodynamic tests were performed in a modified gas gun facility, and the following panel-based examinations of various parameters, such as displacement, velocity, stress, and energy, as well as hydrodynamic ram pressure show that the analysis and test are well correlated, and thus the results of the study reasonably explain the characteristics of the hydrodynamic ram. The methodology and procedures of the present study are applicable to the hydrodynamic ram assessment of airframe survivability design concepts.
\end{abstract}

Key words: Hydrodynamic Ram, Fluid-structure Interaction, Welded joint, Airframe Survivability

\section{Introduction}

This paper addresses the analysis and test of hydrodynamic ram in metallic cubic tanks containing water. Hydrodynamic ram is one of the major man-made threats to aircraft. The effect of hydrodynamic ram is defined as the damage process that a projectile with high velocity impacts a structure with fluid inside, and then penetrates and/or detonates it to produce a blast wave [1]. Among the components of the airframe structure, fuel tanks are more vulnerable to hydrodynamic ram, and especially the wing fuel tanks are most exposed to this ballistic threat because they have large exterior areas.

For this reason, the preparation of fuel tanks against hydrodynamic ram damage is required to meet the structural survivability requirement in the development of an aircraft. Besides fuel pressure exerted on the tank panels, parameters such as fuel volatility, leakage, and joint design are also key factors influencing the scale of resulting structural damage. Thus, survivability design to hydrodynamic ram is not limited to strengthening the tank material or thickness, but also includes features such as fire suppression and advanced joint concepts, too. Foam or a bladder inside the tank, advanced onboard inert gas generation system (OBIGGS), and z-pinning joints are the good examples of such features.

Studies on aircraft battle damage assessment have a long history, but in the early stages, many of them concerned live fire tests of various structures from panel specimens to fullscale airframes [2]. However, it is not practical to test aircraft for all likely scenarios to identify the damage level for each parameter and to assess their weights on the survivability of the whole airframe. Another difficulty is that even if some weakness is found after a live fire test, it is not easy to change
This is an Open Access article distributed under the terms of the Creative Commons Attribution Non-Commercial License (http://creativecommons.org/licenses/bync/3.0/) which permits unrestricted non-commercial use, distribution, and reproduction in any medium, provided the original work is properly cited. (c) * Ph.D. Student

** Professor, Corresponding author: cgkim@kaist.ac.kr *** Director 
the structural design given the fact that live fire test is typically performed in the late development stage where most of the airframe structure is already determined and manufactured, and thus design changes at such a stage put more pressure on the budget and development period.

Hydrodynamic ram is one of the major battle damage threats, and its importance has promoted studies on the understanding of the physics, its prediction, and the influence on the surrounding structures through extensive parametric analyses and tests. Analytical studies have kept pace with the development of fluid-structure interaction theory, analysis techniques, and simulation tools. Some recent examples of aircraft hydrodynamic ram analyses include simulations of aircraft wing penetration and detonation by the present authors [3] and modeling projectile damage in transport aircraft wing structures [4]. Verification tests range from the investigation of hydrodynamic ram parameters, such as the fluid pressure [5], to the study of hydrodynamic ram on a rectangular prism-shaped water tank [6]. Based on these past achievements, the outcomes of the studies on composite structures have been introduced in recent years [7].

Hydrodynamic ram phenomenon is divided fundamentally into three phases: shock, drag, and cavity [1]. Entry or impact is the step that occurs before the shock phase, and the exit is the step that occurs after the cavity phase. Many previous studies identified the occurrence of each phase in hydrodynamic ram tests and properly represented each through simulations. On the other hand, structural behavior has been investigated in less detail compared with the investigation of the fluid phases due to its complexity. However, hydrodynamic ram damage of the airframe component should be more realistically assessed to apply to survivability design, and thus more consideration is required for the investigation. The focus of the present study is on the characteristics of this structural behavior, especially during and after structural failure.

Metallic cubic tanks containing water were used for the test and analysis of hydrodynamic ram. Considering the panels of water tanks were welded to each other, the effect of these welded edges on the structural behavior was investigated as well as the tank material itself. Two cases of different water levels, full and three-quarters full, were also studied to examine the effects of the void.

Arbitrary Lagrange-Euler coupling method was used for the analysis of the fluid-structure interaction occurring in the hydrodynamic ram, and the result was correlated with the test. Panel-based examinations of various engineering parameters were performed for the purpose of gaining a comprehensive understanding of hydrodynamic ram phenomena.

\section{Set-up of Test and Analysis}

\subsection{Summary of Test Preparation}

A cubic tank, made of aluminum, was used for the hydrodynamic ram test. Each side was $406 \mathrm{~mm}$ long and each panel was $2.28 \mathrm{~mm}$ thick. Panels were corner-welded after installing six pressure gauges inside the tank (Fig. 1); three were located along the centerline of the tank with the intention such that the locations would coincide with the traveling path of the projectile, for the purpose of measuring the maximum pressures at each location. The other three were located near the tank wall to measure wall pressures and their influences on the structure.

C300-50-EKRTE carbon pressure gauges (Dynasen Inc.) were used. This gauge measures pressure by resistance change up to $50 \mathrm{ohm}$. It is 3 inches long, but a tiny dotsized carbon element on the aluminum covering is the exact measuring location. The response time of 20ns and a data capture rate of $5 \mathrm{MHz}$ make this gauge adequate for the hydrodynamic ram of very short duration.

Two levels of water - $100 \%$ and $75 \%$ - were used for the test to examine the different interactions and resulting tank behavior of the hydrodynamic ram. A spherical steel ball with a diameter of $19.1 \mathrm{~mm}$ was fired using a modified gas gun to the entry hole of the tank at a planned speed of

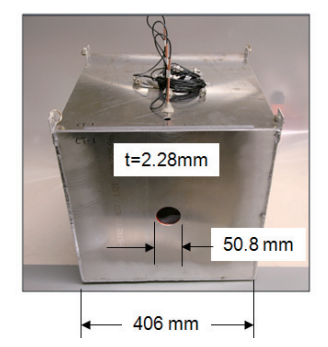

(a) test tank dimensions

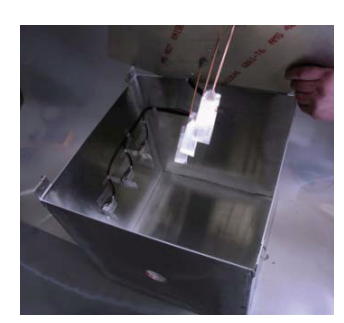

(b) pressure gauges setup

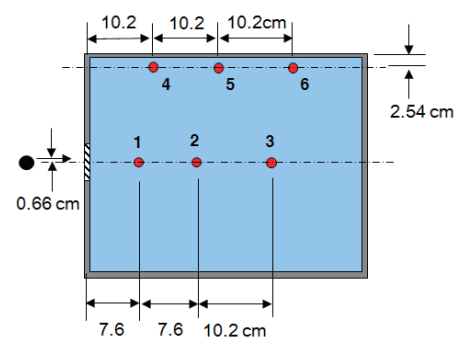

(c) gauge locations

Fig. 1. Test tank preparation 
$914 \mathrm{~m} / \mathrm{s}$, but was measured at $1,000 \mathrm{~m} / \mathrm{s}$ for the fully filled tank and $1,019 \mathrm{~m} / \mathrm{s}$ for the partially filled tank. An entry hole was initially made to guarantee the straightness of the projectile at the entry of the tank. The entry hole was covered with a Mylar film before filling the tank with water to prevent water escape through the entry hole.

\subsection{Summary of Analysis Preparation}

Figure 2 displays the finite element model construction of the hydrodynamic ram. Only half of the model was constructed to take advantage of the symmetry. The model has double symmetry, but only single symmetry was used because in the test results, the travel path of the projectile deviated slightly from the centerline. Fig. 1(c) indicates that the deviation was $6.6 \mathrm{~mm}$ in the partially filled tank case. It was $9.1 \mathrm{~mm}$ for the fully filled tank. This is one of the unavoidable characteristics of gun tests, which ends up influencing the value of peak pressures, especially of the centerline gauges.

The tank is composed of 19,361 shell elements and the water inside the tank, the blue area in Fig. 2, is composed of 259,200 solid elements. There are another 245,800 solid elements outside the tank, the yellow area in Fig. 2, which are initially void for facilitating the water elements coming out of the fractured tank walls during the hydrodynamic ram. The spherical projectile, the red circular area in Fig. 2, has 432 solid elements, and the sizes of those element sides were set such that they were small enough to represent the spherical curve, but not so small compared with the sizes of the water elements to maintain the convergence and precision of the solution.

\section{Analysis Method of Hydrodynamic Ram of Welded Tank}

\subsection{Analysis of fluid-structure interaction}

When there are both structure and fluid in the analysis domain, an analysis with just a Lagrangian formulation that solves the structural constitutive equations of stress and strain of finite elements is not feasible. Due to the

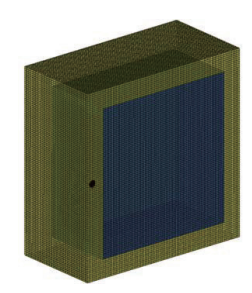

Fig. 2. Model construction of hydrodynamic ram analysis characteristic that the mesh deforms with the material, a Lagrangian formulation is appropriate for the analysis of a model with small deformation, such as a static one. However, if fluid is included in the domain, it creates large deformations, leading to mesh distortion, which makes convergence more difficult and makes the end solution incorrect.

To properly simulate fluid behavior, an Eulerian formulation is required, where elements are fixed in a control volume and the material flows through the mesh. The Eulerian formulation calculates the mass, momentum, and energy conservation equation; that is, the ALE NavierStokes equation [8]. Thus, the final element deformation is determined after the calculation of coupling between the Eulerian and Lagrangian formulations, and this ALE coupling algorithm was used in the present analysis.

While stress-strain equations are required for small volumetric deformations of a structure, an equation of state (EOS) is required once high deformations of fluid occur within the domain. In the fluid, the stress tensor is the sum of the hydrostatic pressure and deviatoric stress. The deviatoric (shear) stress is proportional to the shear strain rate, while EOS defines the hydrostatic pressure behavior.

There are several kinds of EOS and each fluid has its most appropriate EOS, depending on the material and characteristics of the fluid. The equation that can be generally applied is, however, the Gruneisen EOS. In the present analysis, the fluid (water) follows the Gruneisen EOS.

LS-DYNA [9] was used for modeling and computation. After finite element modeling of the projectile, tank, water, and void, as stated in 2.2, the model nodes and elements were brought into LS-DYNA pre-processor. When material data, boundary conditions, and initial conditions were inputted, contact between structures and coupling between structure and fluid are defined. Controlling parameters, such as time step, contact and coupling parameters, and hourglass, also need to be used carefully for the accuracy and convergence of an explicit non linear solution.

\subsection{Analysis of welded joints}

When modeling the finite elements of a structure, the interface between components, such as the panel joint, is typically simplified such that the panels share nodes at the interface, or the interface is assumed to be perfectly bonded. This assumption is valid for a structure with small deformation, because the resulting deformation or strain is heavily dependent on the overall structural material stiffness.

However, for hydrodynamic ram case, where large deformations including interface failure from ram pressure 
occur in a very short period, it is essential to model the interfaces differently because the welded panel joints in the present case now have a strong influence on the overall structural behavior. Indeed, as was found in the test results of the present case, (see Fig. 8, Section 4.2), failure of the weld lines occurred earlier than that of the panel material, and thus, the overall displacement of each panel ended up having considerable rigid body displacement, as well as the structural panel deformation. Observations of the test result that the post-test panels were severely distorted from the combination of these two different kinds of displacement support this argument. The weld lines of the panels failed in the middle of panel bulging, and then the failing panel edges rebounded at separation to produce distorted deformations. This is complex but likely in reality, where there are various interfaces in airframe structures, such as bonded and bolted, and welded joints between components.

The early failure of the weld line comes from the loss of material strength. The material of the test tank was 6061-T6 aluminum alloy and it is highly weldable. The amount of strength decrease depends on the degree of heat treatment, but it is suggested that after welding, the properties near the weld are typically those of annealed 6061-O, which is pretempered and has the lowest strength among 6061 alloys [10]. The Alcoa structural handbook recommends a more conservative strength if used for design [11] because the weld strength in reality varies depending on the amount of heat input, which is different in each case [12].

To add this characteristic in the analysis, weld lines between the tank panels are modeled with the tied interface of LS-DYNA [9]. The tied interface was originally developed to facilitate sudden mesh transitions, where two meshes of surface or solid elements are joined (Fig. 3(a)). This feature can often decrease the amount of effort required to generate meshes because it reduces the need to match nodes across interfaces of merged parts [13].

Although the nodes of the present tank model are not mismatched at the panel interfaces, the panels do not share nodes at the weld lines. The tiebreak contact that comes with

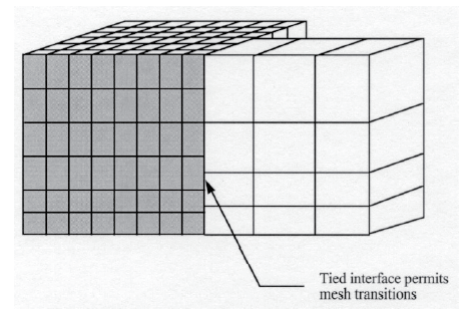

(a) Tied interface for mesh transition

Fig. 3. Modeling of tank panels with weld lines the tied interface is useful for defining the failure criteria of the weld line separately from panel material failure because the tiebreak contact is based on normal and shear strength failure parameters, as indicated in Eq. (1). [14].

$$
\left(\frac{\left|\sigma_{n}\right|}{N F L S}\right)^{2}+\left(\frac{\left|\sigma_{s}\right|}{S F L S}\right)^{2} \geq 1
$$

where $\sigma_{n}(\mathrm{~Pa})$ is the normal tensile stress, $\sigma_{s}(\mathrm{~Pa})$ is the shear stress, NFLS $(\mathrm{Pa})$ is the tensile failure stress, and SFLS (Pa) is the shear failure stress. Thus, the tiebreak contact can facilitate the modeling of connections that transmit both compressive and tensile forces with optional failure criteria. Before failure, the tiebreak contact works both in tension and compression, while after failure, the contact behaves as a surface-to-surface contact with no thickness offsets [15]. In this way, panels have separate nodes with the tiebreak interface, using the property of the weld material, instead of sharing the nodes at the interface even though the mesh is not visually separated. The edges of each panel are separately modeled to represent the weld line and this technique enables early joint failure occurring ahead of the panel material failures, which better represents reality and leads to the greater agreement with the test result.

\section{Investigation of Test and Analysis Results}

\subsection{Pressure history}

Figures 4 and 5 show the pressure versus time measured by three centerline and three wall gauges. Fig. 4 is for the fully filled tank, and Fig. 5 is for the three-quarters-filled tank. Two major pressure peaks of gauge_1 were observed in the graphs of the centerline gauges. The first peak indicates the arrival of the shock wave produced at the impact of the projectile, and the second peak is the arrival of the projectile at the gauge after the gradual pressure increase by the drag. For wall pressure, there are more coupled factors affecting the pressure, such as the pressure wave reflection at the tank

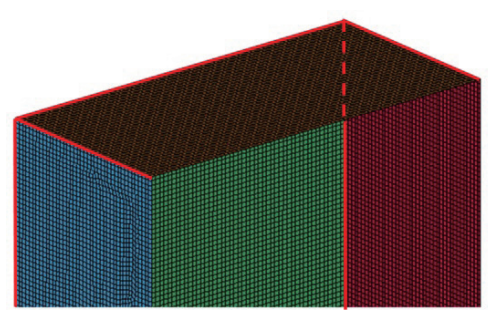

(b) Weld line modeling with tied interface 
panels and the pressure damping by the elasticity of the tank material, which cause higher discrepancies. However, overall, the analysis results follow the trend of the test data.

The differences of the peak value and the corresponding time between the test and analysis are attributed to the characteristics of both the test and analysis. For this kind of very-short-duration test and analysis with high non-linearity, there is a singularity problem, especially for the centerline pressures located on the traveling path of the projectile. The sensitivity of both the test and analysis brings about a large difference even with small input changes.

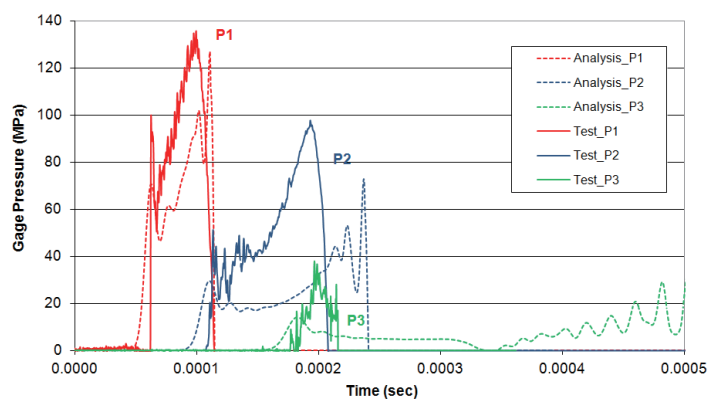

(a) Centerline pressure

Fig. 4. Pressure history by analysis and test for the $100 \%$-filled tank

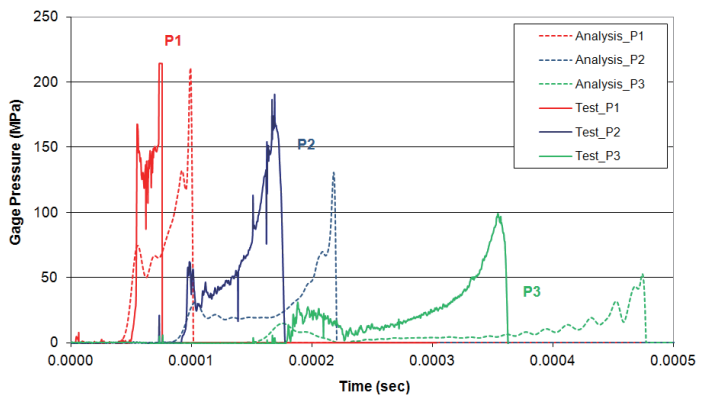

(a) Centerline pressure

Fig. 5. Pressure history by analysis and test for the 75\%-filled tank

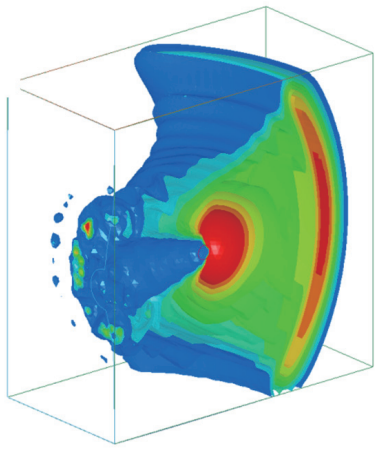

(a) Pressure (MPa) $(\mathrm{t}=0.25 \mathrm{~ms})$
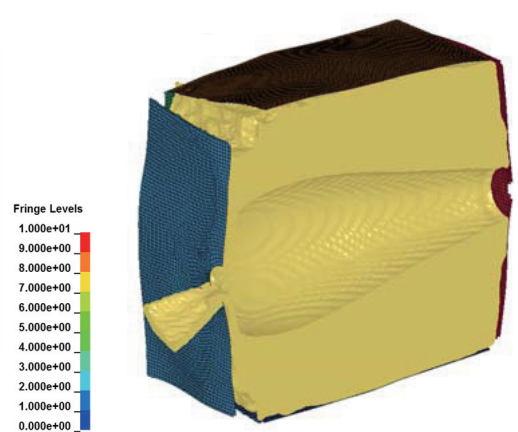

(b) Fluid and structural behavior ( $\mathrm{t}=2 \mathrm{~ms}$ )
If the influence of the different water levels is investigated, the ullage, the void inside the tank, is supposed to lower the drag of the water and thus lower the pressure peaks of center gauges. However, a comparison of the two centerline pressures suggests that the pressures are higher in a short duration for the partially filled tank. These higher pressure peak values of the partially filled tank are actually attributable to a difference in the test conditions. Specifically, first, the projectile entered the tank nearer to the panel center than the fully filled tank case. The distance from the exact hole center to the actual projectile entry was $6.6 \mathrm{~mm}$ for the

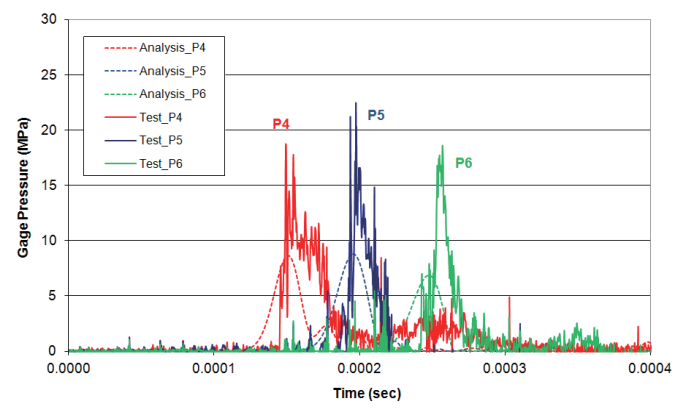

(b) Wall pressure

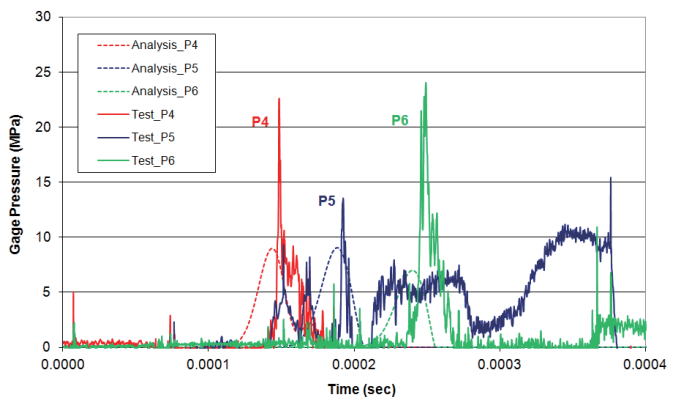

(b) Wall pressure

Fig. 6. Fluid pressure and interaction with structure of the $100 \%$-filled tank panels by analysis 
partially filled tank while it was $9.1 \mathrm{~mm}$ for the fully filled tank. Second, the entry speed at the impact of the projectile to the tank was $1,019 \mathrm{~m} / \mathrm{s}$ for the partially filled tank whereas it was $1,000 \mathrm{~m} / \mathrm{s}$ for the fully filled tank. The differences appear small, but they made a large difference, especially to the pressure peak as the peak, being at the stagnation point, has a singular characteristic.

On the other hand, the overall tendency of the wall pressures does not have as much difference as that of the centerline pressures. It can be inferred that the trend of wall pressures is similar regardless of the different water levels because the wall gauge locations are distant from the projectile path and thus less sensitive than the centerline gauges. If it is considered that the positions (half height) of the wall pressure gauges of the partially (three-quarters) filled tank are below the surface of the water, it can be also inferred that the tendency of the wall pressures will be quite different if the positions are otherwise because the initial conditions at the hydrodynamic ram make a large difference.

The hydrodynamic ram phases are found in the pressure contour of Fig. 6(a). The highest pressure zone, by water drag, is formed right at the front of the projectile, while a cavity is created behind the projectile. The large arc seen up ahead of the projectile near the exit panel signifies the shock wave traveling at the speed of sound, faster than the projectile, which is a typical phenomenon of hydrodynamic ram [16]. The projectile, tank, and water result are shown together in Fig. 6(b), for the visualization of the fluid-structure interaction after the projectile penetrates throughout the tank.

\subsection{Structural deformation and related behavior}

The simulated structural deformation and resulting fracture of the fully filled tank are shown in Fig. 7. The combined effect of fracture at the panel edges and the bulging of the panels by hydrodynamic ram pressure produced distorted panels, especially around the panel edges, which coincided with the test result shown in Fig. 8. The separated panels of the test were attached with duct tape to show the panel distortion compared with the initial state of the tank.

Observation of the deformation and velocity data of each panel in its moving direction at separation enables further understanding of the hydrodynamic ram. Fig. 9 shows that the magnitudes of overall deformation and velocity of each panel are determined by how much the penetrating projectile influences the panel. Also, a closer look at the panel velocities in Fig. 9(b) explains that the velocity curves

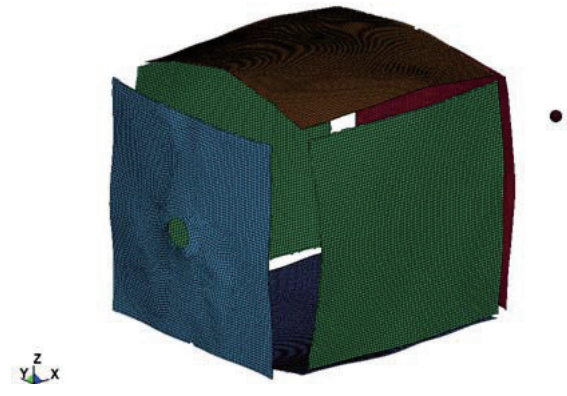

(a) Entry view

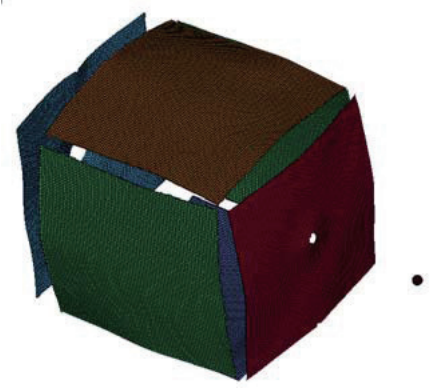

(b) Exit view

Fig. 7. Structural deformation and fracture of the $100 \%$-filled tank panels by analysis

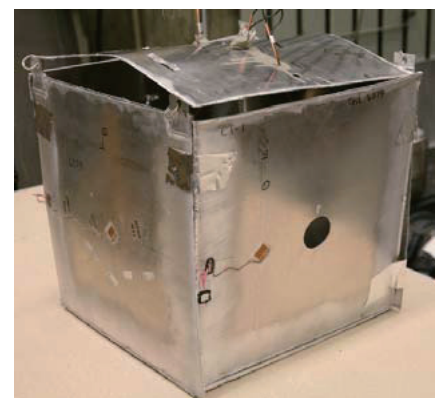

(a) Entry view

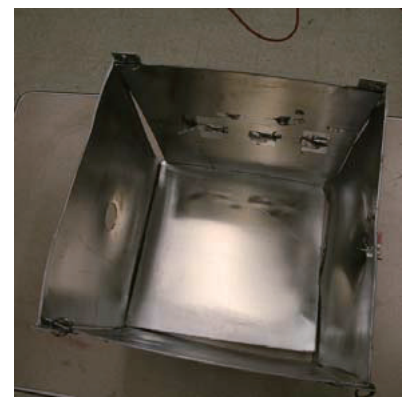

(b) Top view with top panel removed

Fig. 8. Structural deformation and fracture of the $100 \%$-filled tank panels by test 


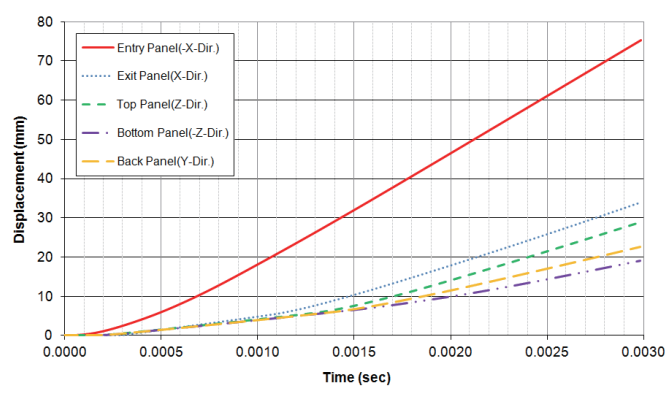

(a) Panel displacement

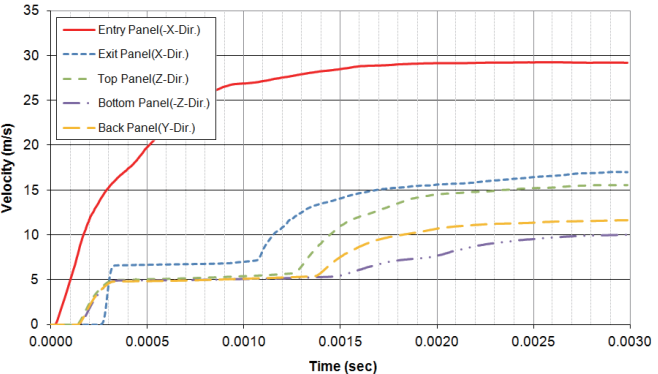

(b) Panel Velocity

Fig. 9. Displacement and velocity of panels for $100 \%$ filled tank by analysis

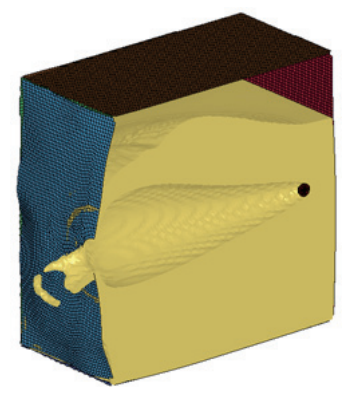

(a) Panel displacement

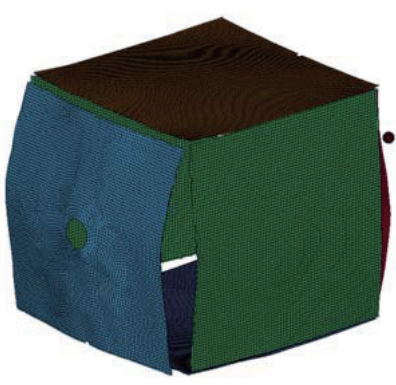

(b) Panel Velocity

Fig. 10. Structural deformation and fracture of the $75 \%$-filled tank panels by analysis

contain the phases of the hydrodynamic ram that occurred during the event. Specifically, for the exit panel velocity, the first small increase, around $0.3 \mathrm{~ms}$, indicates the arrival of the shock wave, and the velocity maintains the same value after the shock wave has gone, and it increases again, after 1.1ms, when the projectile reaches the exit panel and penetrates. The first velocity increases of the top, bottom, and back panels occur earlier than that of the exit panel because they are nearer to the impact location of the entry panel. However, their overall velocity magnitudes are lower than that of the exit panel because they are off the penetrating path of the projectile, from the entry to the exit panel.

Figure 10 shows the structural deformation and fracture of the partially filled tank case. The deformation in the top panel is the lowest among the panels because it needs more time to encounter the rising water. If the deformation versus time data is plotted, it can be observed that the time the top panel starts to deform exactly coincides with the time the water starts to hit the top panel.

\subsection{Momentum and energy}

Figures 11 illustrates the momentum of each panel to the advancing direction of the projectile versus time. Looking at the momentum of the water, the first small peak, at slightly over $0.25 \mathrm{~ms}$, indicates the arrival of the shock pressure at the exit panel, as also witnessed in Fig. 6. The following small valley is produced by the shock pressure completely escaping the exit panel and disappearing. After the shock pressure, the momentum is gradually increased by the traveling projectile pushing the water towards the advancing direction. However, the momentum starts to decrease after $1 \mathrm{~ms}$ because the early failure and separation of the entry panel allow the escape and transfer of the water into the opposite direction, and this opposite momentum overcomes the previous increasing momentum to finally build the later part of the curve.

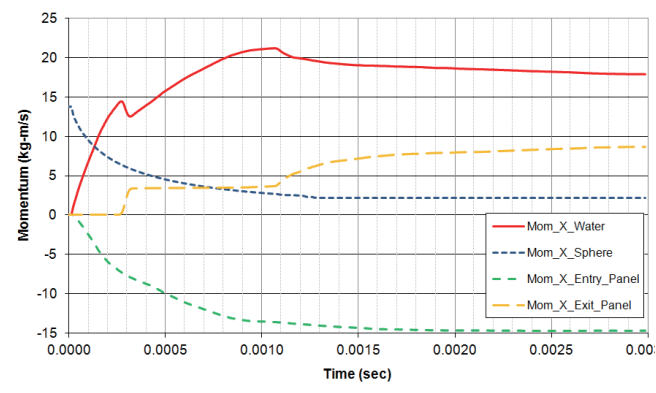

Fig. 11. Momenta of water and panels for the $100 \%$-filled tank 


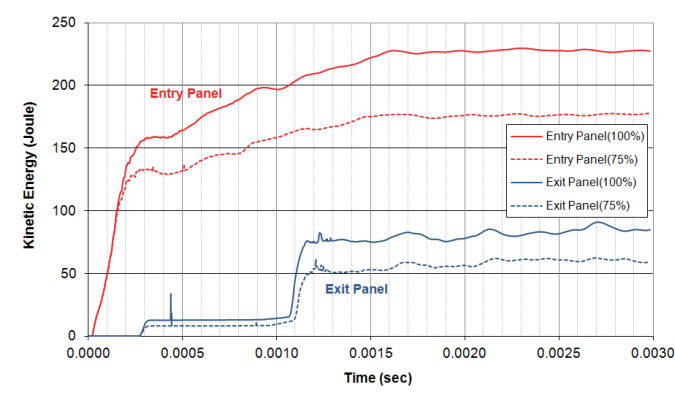

(a) Kinetic energy

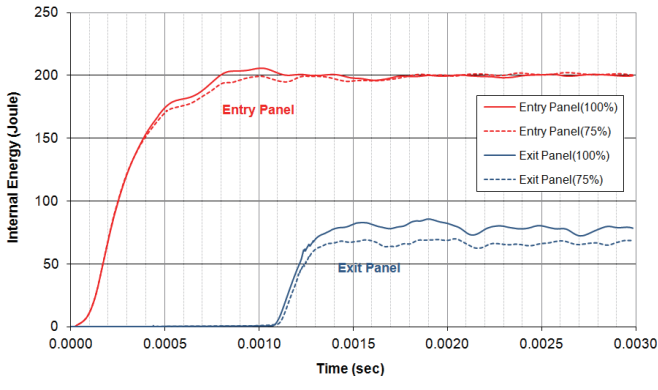

(b) Internal energy

Fig. 12. Energy of panels for $100 \%$ and $75 \%$ filled tanks

On the other hand, the momenta of the panels are mostly attributable to rigid body motions built up since the time they failed completely and separated, and this effect makes the momenta decrease slowly and almost stay at certain values since the time of the separation.

Figure 12 illustrates the kinetic and internal energies of the panels. The data of the entry and exit panels are displayed only for visual clarity. As seen in Fig. 12(a), the kinetic energy of the partially filled tank is lower than that of the fully filled tank because the amount of water, transferring the energy, is less for the partially filled tank. Although it is not illustrated in the figure, the difference the water level makes to the kinetic energy is clearer when investigating the top and bottom panels, because the kinetic energy of the bottom panel is not very different regardless of the water level while the energy of the top panel is quite different, depending on whether the upper void exists or not.

The internal energy of the panels, which signifies how much the panels are deformed, is not much affected by the water level, like the kinetic energy (Fig. 12(b)). The reason for this can be found in Figs. 7 and 10. As compared in the two figures, the entry and exit panels of the partially filled tank are less distorted from less water, but bulge more because the void delays the separation of the panels. These two factors end up building up no less than overall deformations and thus creating the similar energy curve in Fig. 12(b).

\section{Conclusions}

Hydrodynamic ram with welded metallic cubic tanks with water inside was investigated through test and analysis. Two cases of different water levels, fully filled and three-quarters filled, were studied to examine the effects of the void.

The arbitrary Lagrange-Euler coupling method was used for the analysis of the fluid-structure interaction occurring in hydrodynamic ram, where the projectile, tank, and water are exchanging load, momentum, and energy during the traveling of the projectile through the water of the tank. For a better representation of the physical phenomena, modeling of the welded edges was added to the analysis to simulate the earlier weld line fracture and its influence on the resulting hydrodynamic ram behavior.

Panel-based examinations of various parameters, such as displacement, velocity, stress, momentum, and energy were performed, and it showed that the analysis and test correlated well, and thus the result of the study reasonably explained the characteristics of the hydrodynamic ram.

The results showed that the welded joints between tank panels decisively influenced the deformation and failure progress of the tanks. This suggests that the resulting structural damage shape and scale from the hydrodynamic ram, in reality, are highly dependent on the joint mechanism as much as the ram pressures. Thus, ideas for uniform structural robustness against hydrodynamic ram loading should be pursued for survivability designs.

\section{Acknowledgements}

This research was funded by the Agency for Defense Development.

\section{References}

[1] Ball, R., "The Fundamentals of Aircraft Combat Survivability Analysis and Design", AIAA, 2003.

[2] Jacobson, M., "Addendum to Design Manual for Impact Damage Tolerant Aircraft Structure", Aircraft Division of Northrop Corporation, AGARD-AG-238, Hawthorne, CA, 1988.

[3] Kim, J. and Jun, S., "Simulation of Hydrodynamic Ram of Aircraft Fuel Tank by Ballistic Penetration and Detonation", 
International Journal of Modern Physics B, Vol. 22, No. 9-11, 2008, pp. 1525-1530.

[4] Hinrichsen, R., Kurtz, A. and Parks, J., "Modeling Projectile Damage in Transport Aircraft Wing Structures", AIAA, Vol. 46, No. 2, 2008, pp. 328-335.

[5] Hinrichsen, R., Stratton, S., Moussa, A. and Zhang, G., "Hydrodynamic Ram Simulator", Joint Aircraft Survivability Program Report, JASPO-V-07-06-001, Wright-Patterson, OH, 2008.

[6] Varas, D., Lopez-Puente, J. and Zaera, R., "Numerical Analysis of the Hydrodynamic Ram Phenomenon in Aircraft Fuel Tanks", AIAA, Vol. 50, No. 7, 2012, pp. 1621-1630.

[7] Heimbs, S., Nogueira, A., Hombergsmeier, E., May, M. and Wolfrum, J., "Failure Behaviour of Composite T-joints with Novel Metallic Arrow-pin Reinforcement", Composite Structures, 2013, pp. 16-28.

DOI:10.1016/j.compstruct.2013.11.022

[8] Aquelet, N., Souli, M. and Olovsson, L., "Euler-Lagrange Coupling with Damping Effects: Application to Slamming Problems", Computer Methods in Applied Mechanics and Engineering, 2005.
[9] LS-DYNA version 971, Livermore Software Technology Corporation (LSTC), 2007.

[10] 6061 Aluminum Alloy. Available from: http:// en.wikipedia.org/wiki/6061_aluminium_alloy \#Welding.

[11] Alcoa Mill Products, Alcoa Structural Handbook, 2006.

[12] "Guide for Aluminum Welding", Maxal International Inc., 2012, pp. 6-7.

[13] Hallquist, J., LS-DYNA Keyword User's Manual Version 971, Livermore Software Technology Corporation (LSTC), 2007.

[14] McCallum, S., Locking, P. and Harkness, S., "Simulation of Masonry Wall Failure and Debris Scatter", 6th European LS-DYNA Users' Conference, 2007.

[15] Dolce, F., Meo, M., Wright, A. and French, M., "Structural Response of Laminated Composite Plates to Blast Load", 17th International Conference on Composite Materials, Piscataway NJ, 2009.

[16] Disimile, P., Swanson, L. and Toy, N., "The Hydrodynamic Ram Pressure Generated by Spherical Projectiles", International Journal of Impact Engineering, 2009, pp. 821-829. 